\title{
Development and Application of Wireless Power Transmission Systems for Wireless ECG Sensors
}

\author{
Jin-Chul Heo $\mathbb{D}^{1},{ }^{1}$ Jiae Park, ${ }^{1}$ Sohee Kim $\mathbb{D}^{2}{ }^{2}$ Jeonghon Ku, $^{1}$ and Jong-Ha Lee $\mathbb{D}^{1}$ \\ ${ }^{1}$ Department of Biomedical Engineering, School of Medicine, Keimyung University, Daegu 42601, Republic of Korea \\ ${ }^{2}$ Department of Robotics Engineering, Daegu Gyeongbuk Institute of Science \& Technology (DGIST), Daegu 42988, Republic of Korea
}

Correspondence should be addressed to Jong-Ha Lee; segeberg@gmail.com

Received 11 September 2017; Revised 15 November 2017; Accepted 29 November 2017; Published 12 February 2018

Academic Editor: Oleg Lupan

Copyright (C) 2018 Jin-Chul Heo et al. This is an open access article distributed under the Creative Commons Attribution License, which permits unrestricted use, distribution, and reproduction in any medium, provided the original work is properly cited.

\begin{abstract}
We investigated the variations in the magnetic field distribution and power transmission efficiency, resulting from changes in the relative positions of the transmitting and receiving coils, for electromagnetic induction-type wireless power transmission using an elliptical receive coil. Results of simulations using a high-frequency structure simulator were compared to actual measurement results. The simulations showed that the transmission efficiency could be maintained relatively stable even if the alignment between the transmitting and receiving coils was changed to some extent. When the centre of the receiving coil was perfectly aligned with the centre of the transmitting coil, the transmission efficiency was in the maximum; however, the degree of decrease in the transmission efficiency was small even if the centre of the receiving coil moved by $\pm 10 \mathrm{~mm}$ from the centre of the transmitting coil. Therefore, it is expected that the performance of the wireless power transmission system will not be degraded significantly even if perfect alignment is not maintained. Animal experiments confirmed good ECG signals for the simulation conditions. The results suggested a standardized application method of wireless transmission in the utilization of wireless power for implantable sensors.
\end{abstract}

\section{Introduction}

Inductively coupled wireless power transmission, which uses the electromagnetic induction between two coils, is being increasingly applied in medical electronic devices [1, 2]. Recently, the requirement for electronic devices that are much smaller in size than the conventional pacemakers or cochlear implants, such as microneural stimulators or more compact integrated implantable devices for the long-term acquisition of cranial nerve signals, has been increasing [3]. As the size of the implantable smart device becomes smaller, the size of the coil for power transmission must be reduced. The realization of an efficient coil structure within a limited area and volume is thus an important task in wireless power transmission [4].

Although various wireless power transmission technologies have been developed so far, they have not yet been commercialized, except for some noncontact induction coupling methods. In the past, some studies had used microwaves at $5.8 \mathrm{GHz}$ to transmit large powers of several tens of watts or more; however, they have not yet been commercialized actively owing to their effects on the human body and their directivity because of the use of high-efficiency antennas. However, wireless power transmission system is used in the body despite the dangers of electromagnetic waves, and there are a number of development and progress in the medical field $[5,6]$.

Wireless charging using magnetic induction has been performed successfully over short distances of a few millimeters, and it is possible to use a small-sized device of $3 \mathrm{~W}$ or less, and it is known that it can be applied to the human body by using relatively low energy. However, the charging efficiency is extremely low due to the short-receiving distance and a large amount of heat $[7,8]$. The purpose of this study is to develop a wireless sensor that can be used in the human body and its application to wireless electrocardiogram (ECG) 

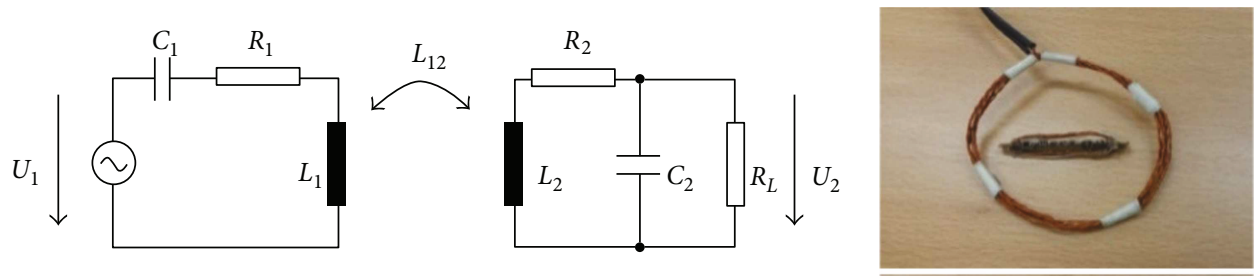

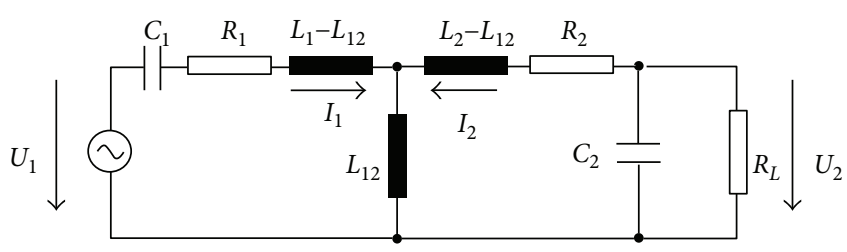

(a)

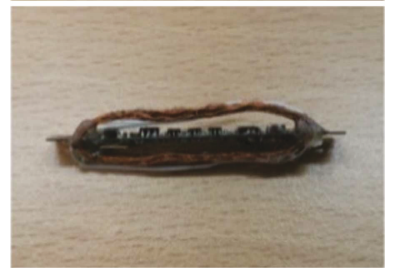

(b)

Figure 1: Wireless power transmission system. (a) Circuit in which the transmit coil $\left(L_{1}\right)$ and the receive coil $\left(L_{2}\right)$ are combined (top), an equivalent circuit of the transmit/receive coil $\left(L_{1}-L_{2}\right)$ (bottom). (b) A circular transmit coil (Tx coil, top) and an elliptical receive coil (Rx coil, bottom).

technology. The wireless ECG sensor is a power supply method by the wireless power transmission through electromagnetic induction between a pair of coils.

In the wireless power transmission system in this study, the two coils generate induced current to transmit power. The transmitting coil and receiving coil form a pair, and the electromotive force induced in the receiving coil by the magnetic field generated when current is supplied to the transmitting coil supplies DC power to the circuit connected to the receiving coil. In this study, we evaluate the theoretical considerations of a wireless power transmission system and the power transmission characteristics, through simulations.

\section{Experiments and Results}

2.1. System Mode. The magnetic field is analysed according to the size and shape of the transmitting and receiving coils. The magnetic field in the gap between the transmitting and receiving coils and the induced current and voltage in the receiving coil are evaluated. For this, computer simulations are performed using the finite element method (FEM). Generally, when using electromagnetic induction for wireless power transmission, the transmit coil $\left(L_{1}\right)$ and receive coil $\left(L_{2}\right)$ both use the capacitance to simulate the $\mathrm{L}-\mathrm{C}$ resonance. A capacitance $C_{1}$ is connected in series to $L_{1}$, and a capacitance $C_{2}$ is connected parallel to $L_{2} . R_{1}$ and $R_{2}$ represent the total resistance in the transmit and receive coils and circuit (Figures 1(a) and 1(b)). The two circuits are magnetically coupled and appear as a coupling coefficient that normalizes the mutual inductance or the mutual inductance to the inductance of each coil. The power transfer efficiency and the power delivered to the load of the receiving circuit can be calculated.

The two circuits are magnetically coupled, and their connection is represented by the mutual inductance $\left(L_{12}\right)$ or the normalized coupling coefficient $(k)$ given by mutual inductance to the inductance of each coil (Figure 1(a)).

$$
k=\frac{L_{12}}{\sqrt{L_{1} L_{2}}} .
$$

In general, the power transfer efficiency $(\eta)$, which is widely used when expressing the power transfer performance, is defined as follows:

$$
\eta=\frac{P_{L}}{P_{S}} .
$$

The power $P_{L}$ transmitted to the load of the receive circuit can be obtained as follows:

$$
P_{L}=\frac{V_{p k}^{2}}{2 R_{L}} .
$$

2.2. FEM Modelling. The high-frequency structure simulator (HFSS) of ANSYS Company, which is a specialized software for the finite element analysis of electromagnetic fields, is used. The HFSS can be used to simulate electromagnetic fields and electronic circuits in all frequency domains; hence, it is possible to simulate the electromagnetic field generated by the coil even when a coil for radio power transmission is connected to the $\mathrm{L}-\mathrm{C}$ resonance circuit. In the simulations, the phenomenon, wherein the transmit and receive coils resonate at $13.56 \mathrm{MHz}$, and the distributions of the magnetic fields, formed inside the transmit and receive coils, are examined. In addition, when a specific current is inputted to the transmit coil, the distance between the transmit and receive coils and the alignment between the transmit and receive coils are used to evaluate the voltage and electric power in the receive coil. The conditions used in the simulations are as follows:

(1) A wire with a circular cross-sectional diameter of $0.5 \mathrm{~mm}$ requires a long time for the generation of meshes and simulations for the FEM. The circular cross-section can therefore be converted to a rectangular cross-section with the same area, to model the wire as a rectangle of $0.44 \mathrm{~mm}$ length. 


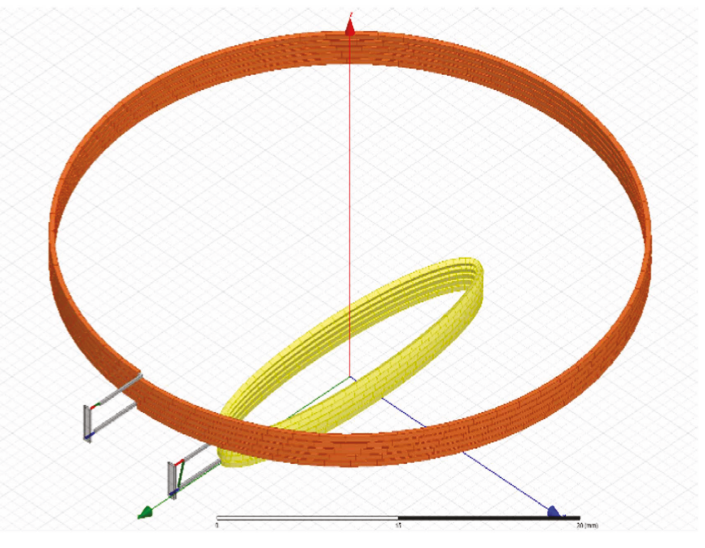

(a)

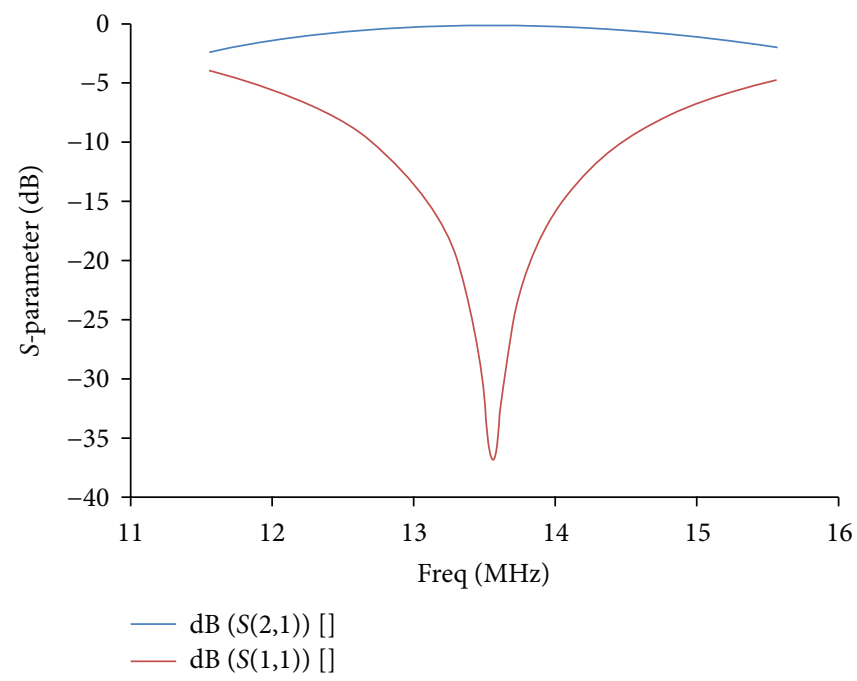

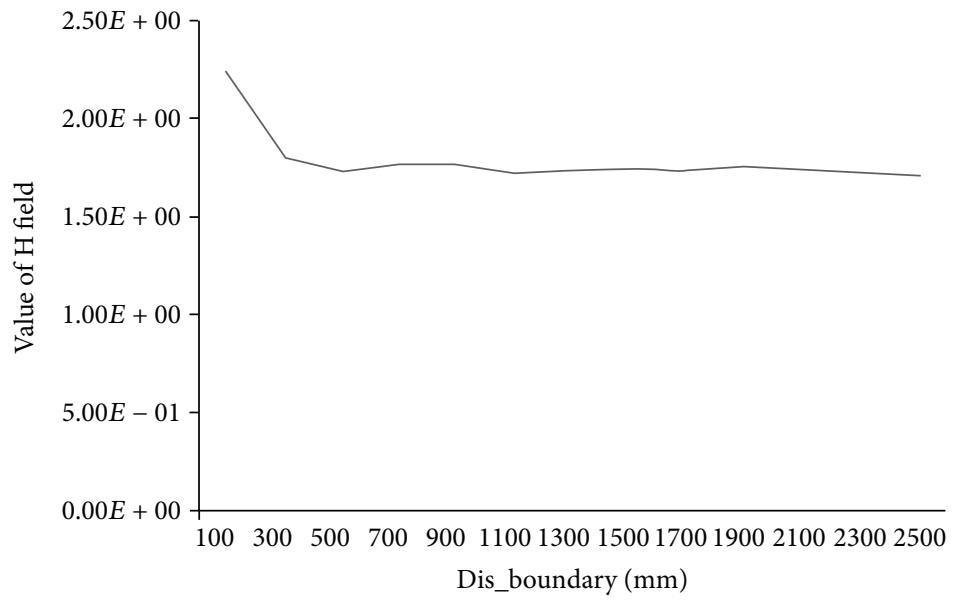

(b)

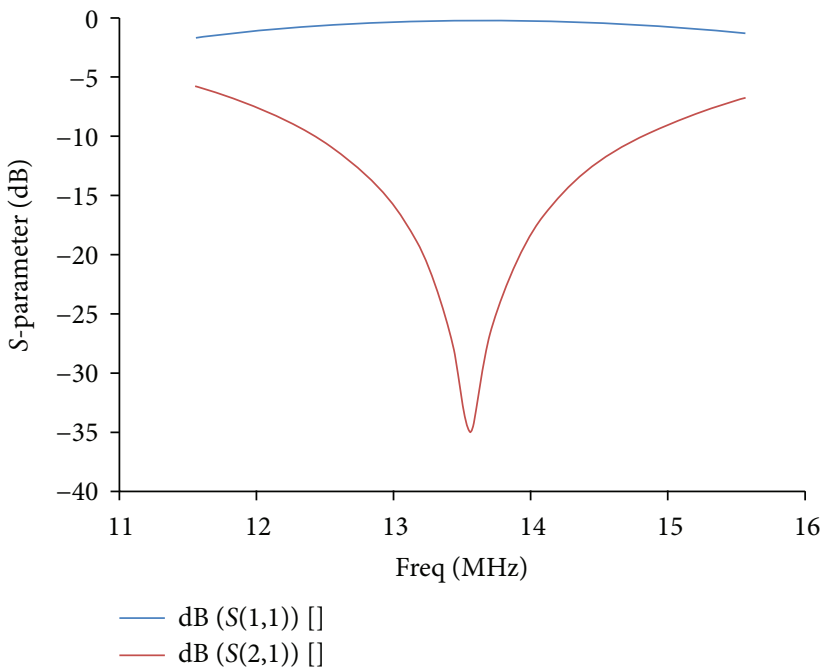

(c)

Figure 2: (a) Modelling transmit coil (orange) and receive coil (yellow). (b) Convergence test for the magnetic field strength when the size of the system simulation is increased. (c) Resonance confirmation at $13.56 \mathrm{MHz}$ for transmit (orange) coil (left) and receive (yellow) coil (right).

(2) The transmit coil (Tx coil) is modelled 5th winding form with a $5 \mathrm{~cm}$ round coil, and the receive coil ( $\mathrm{Rx}$ coil) is modelled 5th winding by an elliptical coil with a major axis of $3 \mathrm{~cm}$ and a minor axis of $0.7 \mathrm{~cm}$. In the figure below, orange represents the transmit coil and yellow the receive coil (Figure 2(a)).

(3) While determining the size of the space for simulation, increasing the boundary of the magnetic field intensity and inductance value at a specific position, so that even if the boundary is increased, there is no change in the value of the magnetic field strength and inductance. The convergence point is set to the optimal boundary satisfying both efficiency and accuracy. As shown in the following figures, the size of $500 \mathrm{~mm}$ is determined suitable for the total system size to be simulated (Figure 2(b)).
(4) A two-port model of the selectable options in HFSS was adopted to add a capacitor to resonate at 13.56 MHz with both the transmit and receive coils. After calculating the resonance capacitance from $f=1 / 2 \pi \sqrt{L C}$ to $C=1 /(2 \pi f)^{2} L$, the capacitance value was changed slightly based on the calculated value, and the exact capacitance value was found for the desired resonance frequency.

When a specific current value is inputted to the transmit coil, the distance between the transmit and receive coils and the alignment between the transmit and receive coils are used to evaluate the voltage and electric power in the receive coil (Figure 2(c)).

2.3. Simulation and Verification of ECG Sensor. The distribution of the magnetic field (H-field) when an AC current at $13.56 \mathrm{MHz}$ is applied to the transmit coil and the 

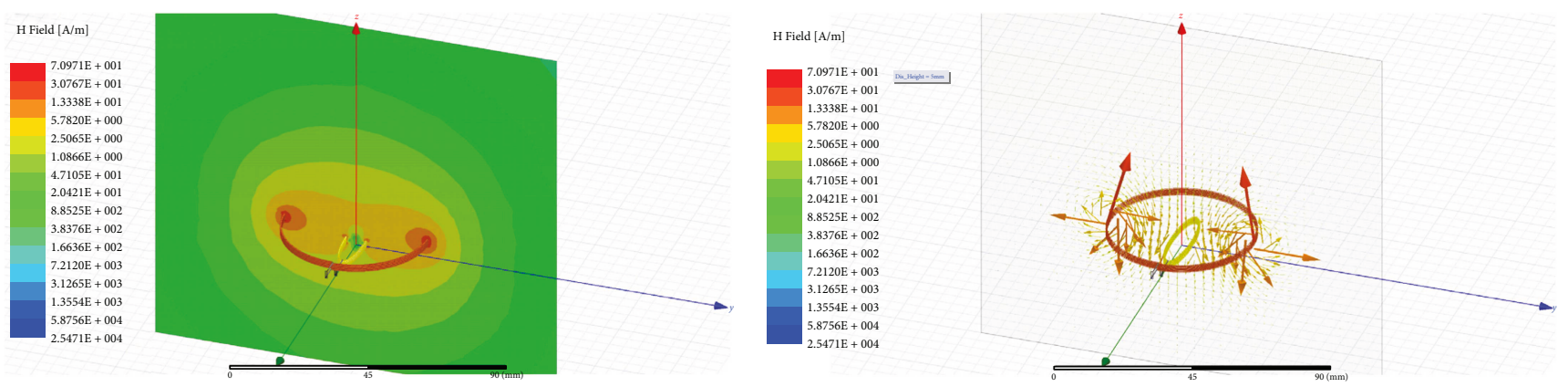

(a)
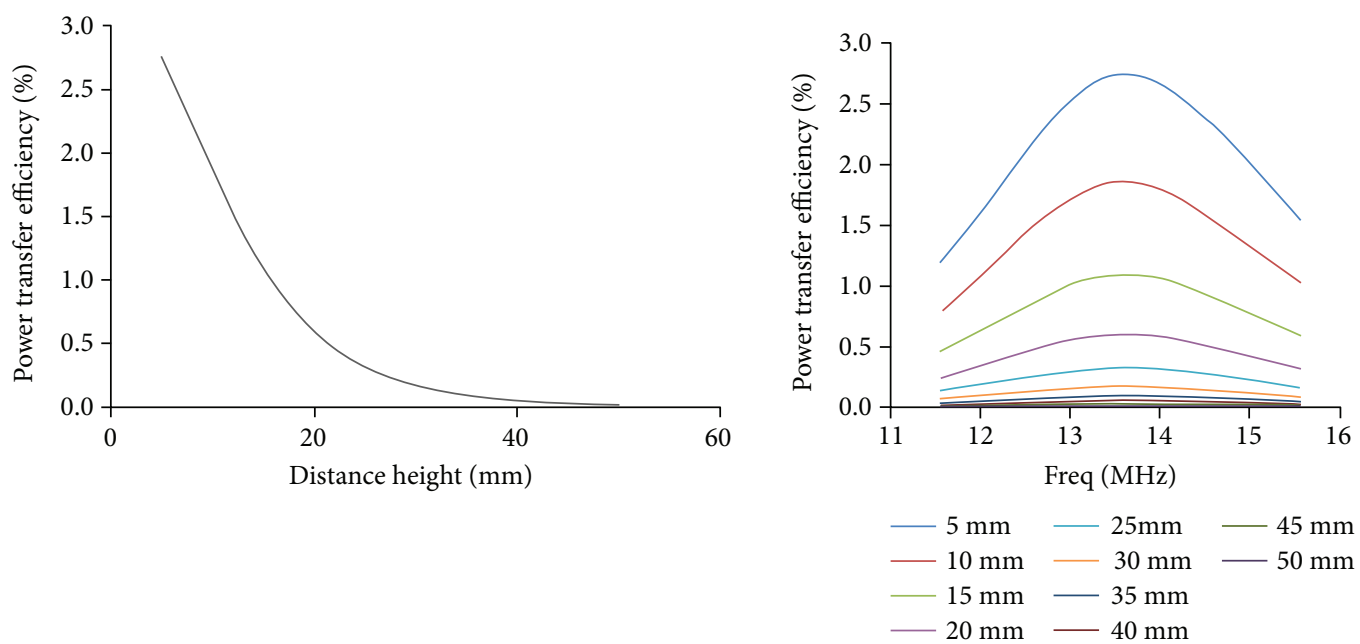

(b)

Figure 3: (a) Distribution of magnetic field formed when $13.56 \mathrm{MHz}$ alternating current flows through the transmitting coil: (left) contour plot, (right) vector plot. (b) Changes in power transmission efficiency with increase in distance between transmit and receive coils (left) and frequency (right).

receive coil is located at a certain distance away from the $z$-axis. The results of calculating the power transmission efficiency according to the relative positions of the transmit/receive coils as well as the magnetic field formed are shown in Figure 3(a).

The change in the magnitude of the magnetic field in the case where the centres of the receive coil and transmit coil are perfectly aligned (misalignment is zero); the transmission coil is shifted $5 \mathrm{~mm}$ in the major axis direction of the ellipse until it becomes $25 \mathrm{~mm}$. The change in the power transmission efficiency when the receive coil is moved in the major axis direction and the alignment turn aside. At this time, the distance between the transmit and receive coils in the $z$ axis direction is fixed to $5 \mathrm{~mm}$. In a perfectly aligned state, the transmission efficiency is approximately $1.85 \%$, and the transmission efficiency is decreased as the degree of misalignment increases. However, there is no significant difference in the transmission efficiency until the alignment is changed by approximately $10 \mathrm{~mm}$ (Figure 3(b)).

The change in the magnetic field when the alignment is changed by moving the transmit coil by $0 \mathrm{~mm}$ to $25 \mathrm{~mm}$ in the minor axis direction of the ellipse is shown in Figure 4(a). There is a change in the power transfer efficiency when the receive coil is moved in the uniaxial direction. The distance in the $z$-axis direction between the transmit and receive coils is fixed at $5 \mathrm{~mm}$, and the transmission efficiency is the highest in a perfectly aligned state. As the degree of misalignment increases, the transmission efficiency should decrease. However, it can be seen that there is no significant difference in the transmission efficiency (Figure 4(b)).

AC current at $13.56 \mathrm{MHz}$ is supplied to the transmit coil, and the distributions of the magnetic field are observed when the receive coil is located at a certain distance away from the $z$-axis. When the distance between the coils is $5 \mathrm{~mm}$, the transmission efficiency is approximately $2.74 \%$. The maximum transmission efficiency is obtained at the resonance frequency of the transmit/receive coil, and the transmission efficiency is lower at frequencies other than the resonance frequency. There is a change in the magnitude of the magnetic field in the case where the centres of the receive coil and transmit coil are perfectly aligned (misalignment is zero). The transmit coil is shifted $5 \mathrm{~mm}$ in the long-axis direction of the ellipse until it becomes shifted by $25 \mathrm{~mm}$. The change in the power transmission efficiency when the receive coil is moved along the long-axis and short-axis directions and the alignment turn aside. At this time, the distance between the transmit and receive coils in the $z$-axis direction is fixed at $5 \mathrm{~mm}$. In a perfectly aligned state, the transmission efficiency is approximately 


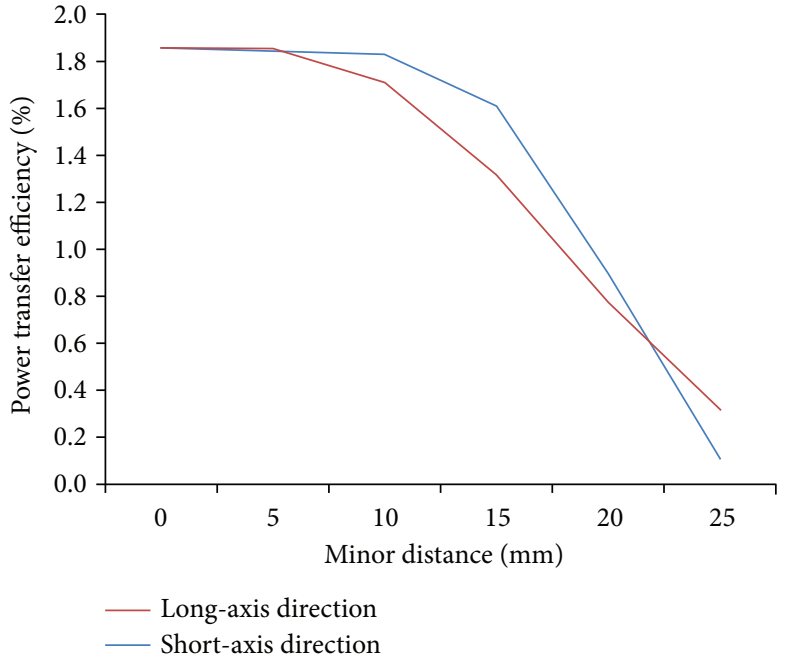

(a)

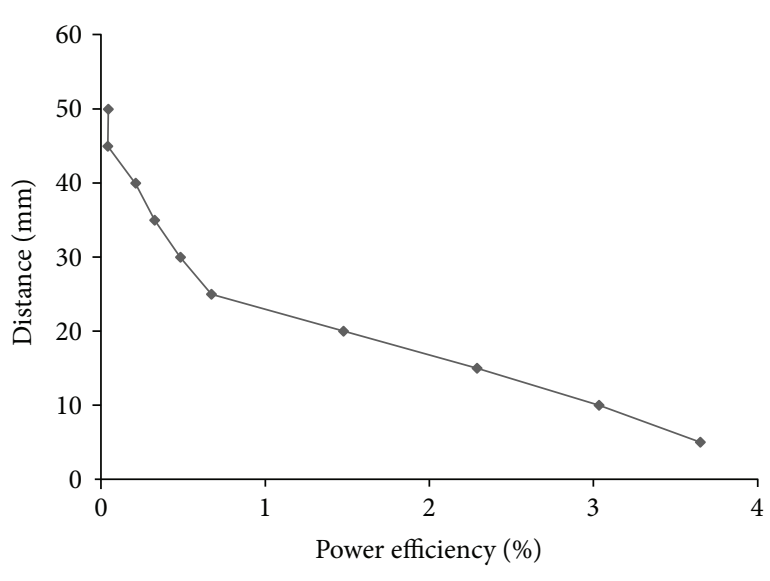

(b)

Figure 4: (a) When the alignment between the transmit coil and receive coil is different in the long-axis/short-axis direction of the receive coil. (b) The power transmission efficiency.

TABle 1: Power transmission efficiency.

\begin{tabular}{lcccccc}
\hline Distance $(\mathrm{mm})$ & $V_{\mathrm{p}}(\mathrm{V})$ & $\mathrm{VR}_{-} \mathrm{Tx}(\mathrm{V})$ & $I_{\mathrm{s}}(\mathrm{A})$ & $P_{\mathrm{s}}(\mathrm{W})$ & $P_{\mathrm{L}}(\mathrm{W})$ & Power efficiency $(\%)$ \\
\hline 5 & 7.3 & 1.46 & 0.146 & 0.73 & 0.026645 & 3.65 \\
10 & 6.7 & 1.48 & 0.148 & 0.74 & 0.022445 & 3.033108108 \\
15 & 5.9 & 1.52 & 0.152 & 0.76 & 0.017405 & 2.290131579 \\
20 & 4.8 & 1.56 & 0.156 & 0.78 & 0.01152 & 0.476923077 \\
25 & 3.28 & 1.6 & 0.16 & 0.8 & 0.005379 & 0.6724 \\
30 & 2.8 & 1.62 & 0.162 & 0.81 & 0.00392 & 0.483950617 \\
35 & 2.3 & 1.62 & 0.162 & 0.81 & 0.002645 & 0.32654321 \\
40 & 1.84 & 1.6 & 0.16 & 0.8 & 0.001693 & 0.2116 \\
45 & 0.82 & 1.62 & 0.162 & 0.81 & 0.000336 & 0.041506173 \\
50 & 0.85 & 1.62 & 0.162 & 0.81 & 0.000361 & 0.044598765 \\
\hline
\end{tabular}

$1.85 \%$, and the transmission efficiency should decrease as the degree of misalignment increases. However, there is no significant difference in the transmission efficiency until the alignment is changed by approximately $10 \mathrm{~mm}$. The transmit coil has the highest transmission efficiency in the perfectly aligned state, because of the change in the magnetic field when the alignment is changed by moving from $0 \mathrm{~mm}$ to $25 \mathrm{~mm}$ in the minor axis direction of the ellipse. It can be seen that there is no significant difference in the transmission efficiency until the alignment is shifted by approximately $15 \mathrm{~mm}$.

In order to verify the wireless power transmission observed in the simulations, we performed actual experiments. In the experiments, a circular transmit coil with a diameter of $5 \mathrm{~cm}$ and an elliptical receive coil with a long axis/short axis of $30 \mathrm{~mm} / 7 \mathrm{~mm}$ were fabricated. Both the transmit and receive coils were coated with copper wire having a diameter of $0.5 \mathrm{~mm}$, and the number of turns of the coils was set to 5 . The inductance of the fabricated coil was measured as $2.8 \mu \mathrm{H}$ for the transmit coil and $0.6 \mu \mathrm{H}$ for the receive coil. The resonant capacitance used to resonate the transmit and receive coils at $13.56 \mathrm{MHz}$ was $37 \mathrm{pF}$ and $228 \mathrm{pF}$, respectively (Table 1 ).

When the distance between the transmit and receive coils was $5 \mathrm{~mm}$, the voltage of the receive coil (using $1 \mathrm{k} \Omega$ ) was $7.3 \mathrm{~V}$, the power was $26.6 \mathrm{~mW}$, and the power transmission efficiency was $3.6 \%$. When the distance between the coils was $20 \mathrm{~mm}$, the voltage, power, and transmission efficiency at the receive coil were $4.8 \mathrm{~V}, 11.5 \mathrm{~mW}$, and $1.5 \%$, respectively. If the actual load (IC chip of various amplifiers), to which the receive coil will transmit power, requires $3.3 \mathrm{~V} \mathrm{DC}$ operating power, when the input exceeds $4.8 \mathrm{~V}$, the $3.3 \mathrm{~V}$ DC voltage can be generated sufficiently if a full-wave rectifier is used to convert the induced AC voltage to $\mathrm{DC}$ voltage. In other words, if we use the same coils as those used in this measurement, the transmit and receive coils will be able to supply enough voltage and power even when they are $2 \mathrm{~cm}$ apart.

An experiment in which the voltage value obtained at the receive coil is measured while the distance between the coils 

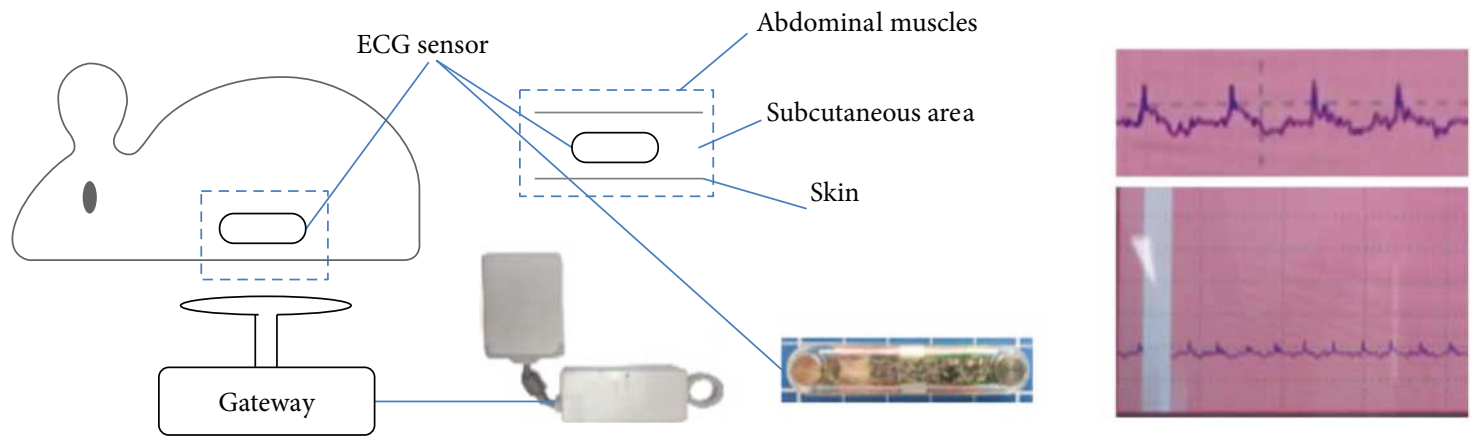

FIGURE 5: ECG sensor verification using in vivo model and wireless signal transmission.

is changed, while maintaining the input to the transmit coil constant using the transmit/receive coil, was conducted. The results of measuring the voltage and power were obtained from the receive coil according to the distance between the coils. The power transmission efficiency was calculated as described in (2) and (3) (Figure 4(b)):

Transmission efficiency $(\eta(\%))=(\mathrm{PL} / \mathrm{PS}) * 100$.

Power at the load of the receiving coil $(\mathrm{PL})=($ "Vpk $) 2 " /$

2RL.

Power in the transmit coil $(\mathrm{Ps})=\mathrm{Vs}{ }^{*}$ Is.

2.4. In Vivo Model Validation. Fifteen healthy adult male Sprague-Dawley rats weighing 380-460 g were implanted with ECG sensors, which were activated. After inserting sensors between the peritoneal epithelium and the skin, the surgical site was allowed to heal for a period of approximately 4 weeks. The experiments were performed in accordance with the guidelines outlined in the Declaration of Helsinki and were approved by the Ethic Committee of Keimyung University (Approval number, KM-2015-20R1). The wireless power system received signals from the sensor containing the ECG electric power supply, to transmit to an external monitor via Bluetooth. The display system consisted of an ECG signal output using a smartphone (Figure 5). The transplanted ECG sensor showed a normal signal and confirmed that the experimental animal showed a pattern similar, less than $5 \%$ difference, to the simulation result of the wireless transmission in the fixed state.

\section{Discussion}

As the population ages and the welfare is increased, studies on the implantable medical devices are actively being carried out, and products providing more various functions are being released. On the other hand, the power consumption of the human implantable medical device is increasing due to the various functions, and thus the primary battery alone does not provide sufficient power. This study investigated the efficiency of wireless power transmission system using magnetic induction method. We confirmed the efficiency of ECG sensor power transmission through computer modeling and applied it to an animal model using a rat to check the applicability of the human body using a magnetic induction wireless power transmission system. As the required functions of the implantable device are diversified, the power use time is reduced, and the cycle of reoperation for power source replacement is shortened, so that additional cost burden and physical and psychological burdens are increased. The development of a sustainable power module that reduces the cost and psychological burden of these additional surgeries is an indispensable elemental technology that enables more functional implementation of medical devices for human implantation.

Recently, the human implantable medical device market is rapidly growing due to population aging and welfare policies. Implantable medical devices have been increasingly applied to various disease treatment fields in order to assist human weak functions, and power modules have become increasingly necessary for active treatment through devices. As the wireless power transmission technology has been actively studied, many attempts have been made to apply a method using an electromagnetic wave such as electromagnetic induction technology or magnetic resonance method commercialized in an electronic product to a human body insertion device. However, the low-frequency electromagnetic wave has a low absorption rate in the human body, the transmission distance is short, and electromagnetic waves of high frequency have a large absorption rate in the human body, which causes a rise in temperature of skin tissues and which limits the application $[9,10]$.

The magnetic induction type wireless transmission system used in this study receives electric induction between the transmitter coil and the receiver coil. When a magnetic field is generated in the transmitter coil, the receiver coil receives the magnetic field and induces the electric power. In this method, the transmission efficiency is as high as $90 \%$ or more, but the transmission distance is very short as several millimeters, and if the centres of the coils are not aligned with each other, the transmission efficiency is greatly reduced. However, it is known that the safety and efficiency compared to the magnetic resonance method and the electromagnetic wave method are the most suitable to be applied to the medical field so far. The results of this study can be used as a model for the power transmission system such as human transplantation sensor using the magnetic induction method. A limitation of the present study did not reflect the movement of animals for in vivo transplantation. In order to efficiently apply the results of this study, it is necessary to verify the wireless power transmission efficiency for sensor movement. 


\section{Conclusion}

In the electromagnetic induction-type wireless power transmission, the magnetic field distribution according to the relative positions of the transmit and receive coils and the power transmission efficiency are very important. The results of HFSS simulations using the ECG sensor were compared with the actual measurement results. If the distance between the coils was maintained less than $2 \mathrm{~cm}$, the voltage that the receive coil could deliver was more than $4.8 \mathrm{~V}$, and the transmission efficiency was approximately $1.5 \%$. Simulations showed that the transmission efficiency could be maintained relatively stable, regardless of the degree of alignment between the transmit and receive coils; the results were verified using an animal model.

\section{Conflicts of Interest}

The authors declare that there are no conflicts of interest regarding the publication of this paper.

\section{Acknowledgments}

This research was supported by the Keimyung University Research Grant of 2017.

\section{References}

[1] B. Lee, M. Kiani, and M. Ghovanloo, "A triple-loop inductive power transmission system for biomedical applications," IEEE Transactions on Biomedical Circuits and Systems, vol. 10, no. 1, pp. 138-148, 2016.

[2] H. G. Lim, J. H. Kim, D. H. Shin et al., "Wireless charing pillow for a fully implantable hearing aid: design of a circular array coil based on finite element analysis for reducing magnetic weak zones," Bio-Medical Materials and Engineering, vol. 26, no. s1, pp. S1741-S1747, 2015.

[3] M. D. Ker, C. Y. Lin, and W. L. Chen, "Stimulus driver for epilepsy seizure suppression with adaptive loading impedance," Journal of Neural Engineering, vol. 8, no. 6, article 066008, 2011.

[4] S. E. Jo, S. Joung, J. K. Suh, and Y. J. Kim, "Improvement of wireless power transmission efficiency of implantable subcutaneous devices by closed magnetic circuit mechanism," Medical \& Biological Engineering \& Computing, vol. 50, no. 9, pp. 973980, 2012.

[5] K. M. Thotahewa, J. M. Redoute, and M. R. Yuce, "Electromagnetic and thermal effects of IR-UWB wireless implant systems on the human head," in Conference proceedings : Annual International Conference of the IEEE Engineering in Medicine and Biology Society IEEE Engineering in Medicine and Biology Society Annual Conference, vol. 2013, pp. 5179-5182, Osaka, Japan, 2013.

[6] N. Carranza, V. Febles, J. A. Hernandez et al., "Patient safety and electromagnetic protection: a review," Health Physics, vol. 100, no. 5, pp. 530-541, 2011.

[7] B. Swain, P. P. Nayak, D. P. Kar, S. Bhuyan, and L. P. Mishra, "Wireless energizing system for an automated implantable sensor," Review of Scientific Instruments, vol. 87, no. 7, article 074708, 2016.
[8] M. Kiani, U. M. Jow, and M. Ghovanloo, "Design and optimization of a 3-coil inductive link for efficient wireless power transmission," IEEE Transactions on Biomedical Circuits and Systems, vol. 5, no. 6, pp. 579-591, 2011.

[9] K. R. Foster and R. Glaser, "Thermal mechanisms of interaction of radiofrequency energy with biological systems with relevance to exposure guidelines," Health Physics, vol. 92, no. 6, pp. 609-620, 2007.

[10] H. P. Schwan and G. M. Piersol, "The absorption of electromagnetic energy in body tissues: A review and critical analysis," American Journal of Physical Medicine \& Rehabilitation, vol. 33, no. 6, pp. 371-404, 1954. 


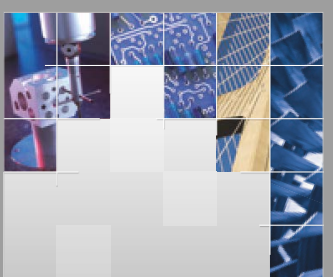

\section{Enfincering}
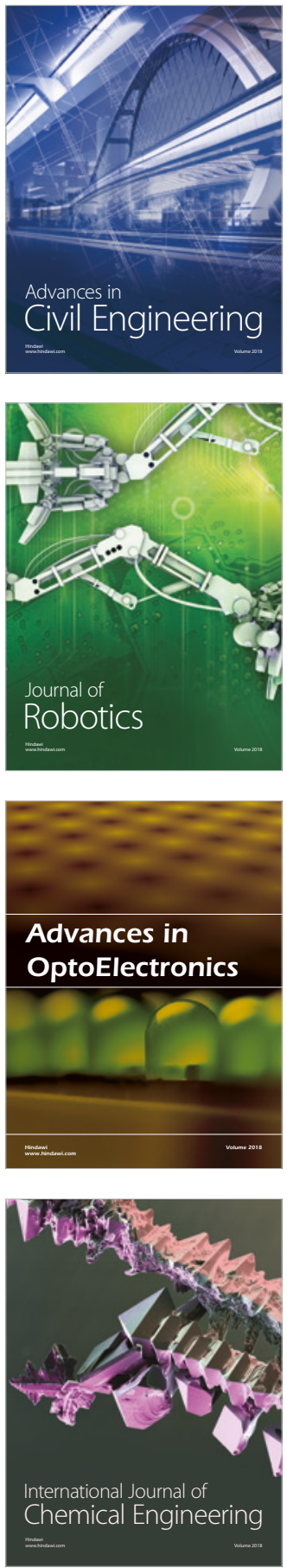

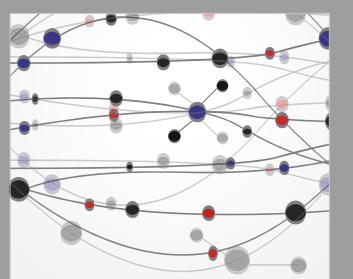

\section{Rotating \\ Machinery}

The Scientific World Journal

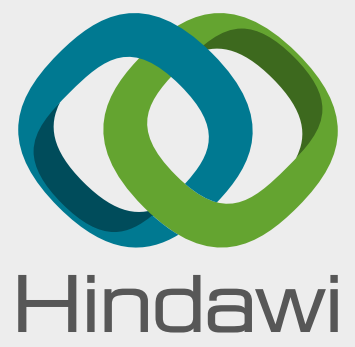

Submit your manuscripts at

www.hindawi.com
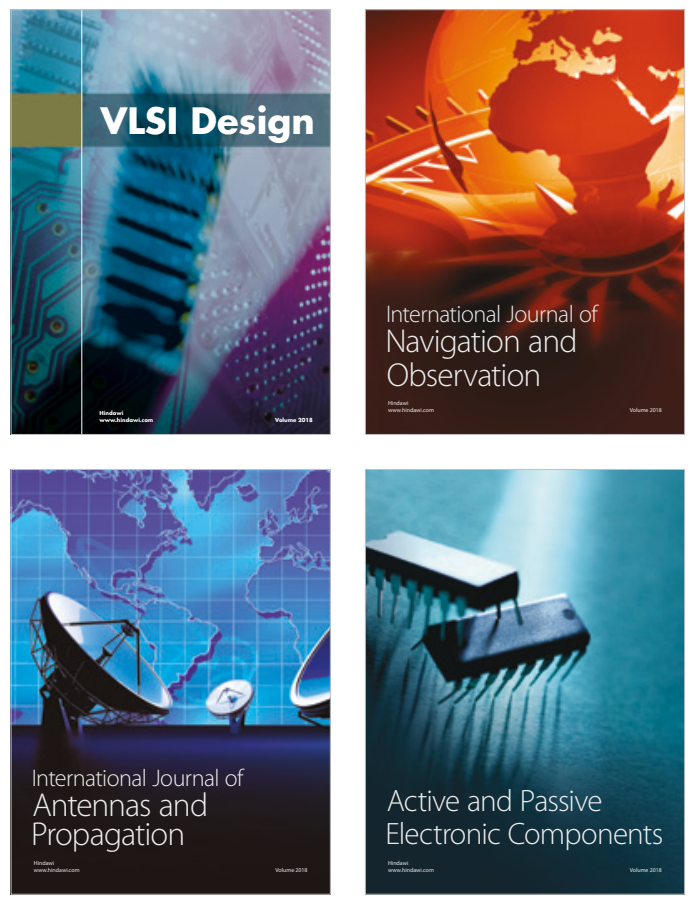
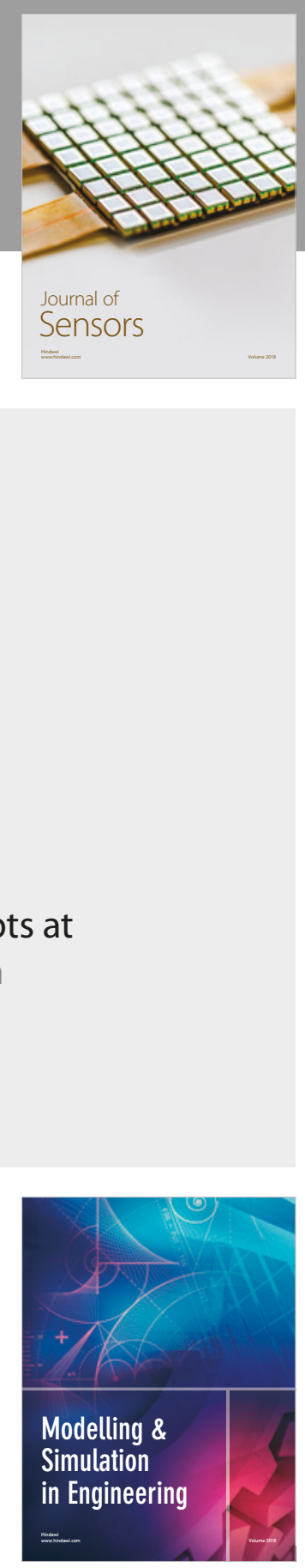

\section{Advances \\ Multimedia}
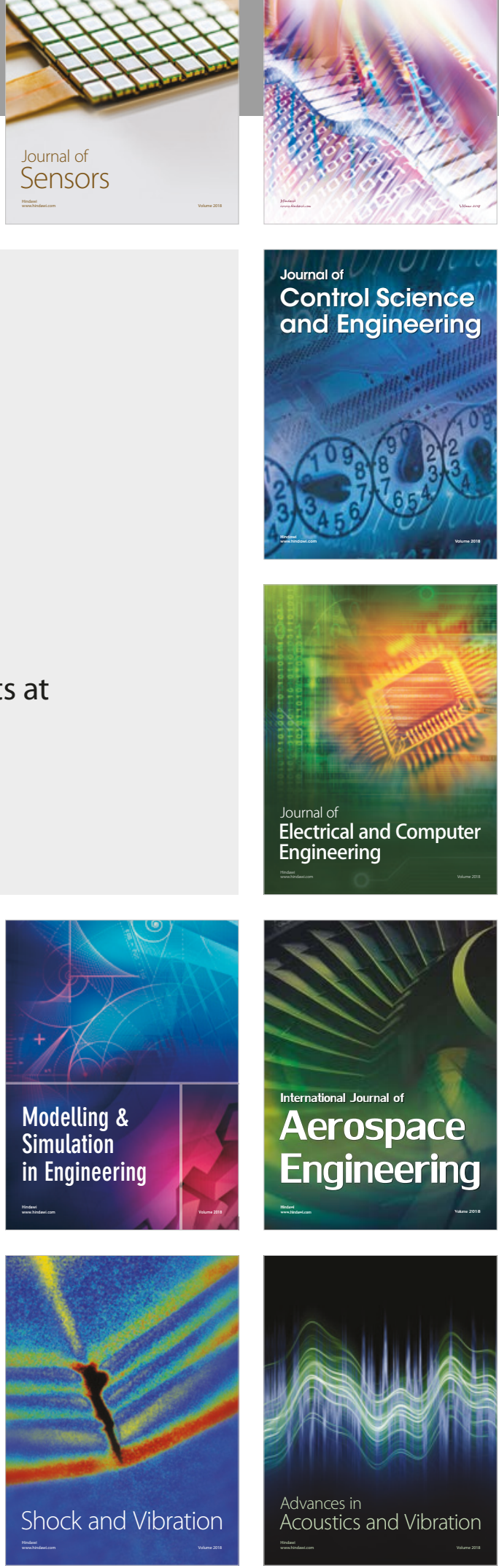